\title{
A Novel Method for Lithium-Ion Battery Online Parameter Identification Based on Variable Forgetting Factor Recursive Least Squares
}

\author{
Zizhou Lao ${ }^{1}$, Bizhong Xia ${ }^{1, *}$, Wei Wang ${ }^{2}$, Wei Sun ${ }^{2}$, Yongzhi Lai ${ }^{2}$ and Mingwang Wang ${ }^{2}$ \\ 1 Graduate School at Shenzhen, Tsinghua University, Shenzhen 518055, China; 1zz15@mails.tsinghua.edu.cn \\ 2 Sunwoda Electronic Co., Ltd., Shenzhen 518108, China; yuhanbo@sunwoda.com (W.W.); \\ sunwei@sunwoda.com (W.S.); lyz@sunwoda.com (Y.L.); yetongzhou@sunwoda.com (M.W.) \\ * Correspondence: xiabz@sz.tsinghua.edu.cn; Tel.: +86-180-3815-3128
}

Received: 2 May 2018; Accepted: 24 May 2018; Published: 26 May 2018

\begin{abstract}
For model-based state of charge (SOC) estimation methods, the battery model parameters change with temperature, SOC, and so forth, causing the estimation error to increase. Constantly updating model parameters during battery operation, also known as online parameter identification, can effectively solve this problem. In this paper, a lithium-ion battery is modeled using the Thevenin model. A variable forgetting factor (VFF) strategy is introduced to improve forgetting factor recursive least squares (FFRLS) to variable forgetting factor recursive least squares (VFF-RLS). A novel method based on VFF-RLS for the online identification of the Thevenin model is proposed. Experiments verified that VFF-RLS gives more stable online parameter identification results than FFRLS. Combined with an unscented Kalman filter (UKF) algorithm, a joint algorithm named VFF-RLS-UKF is proposed for SOC estimation. In a variable-temperature environment, a battery SOC estimation experiment was performed using the joint algorithm. The average error of the SOC estimation was as low as $0.595 \%$ in some experiments. Experiments showed that VFF-RLS can effectively track the changes in model parameters. The joint algorithm improved the SOC estimation accuracy compared to the method with the fixed forgetting factor.
\end{abstract}

Keywords: variable forgetting factor; recursive least squares; lithium-ion battery; online parameter identification; state of charge

\section{Introduction}

Compared with other batteries, the performance of lithium-ion batteries is better in terms of power capability, cycle life, thermal stability, and so forth [1]. Therefore, the lithium-ion battery industry has developed rapidly, and the batteries have a wide range of commercial applications, such as in electric vehicles, cell phones, laptop aviation products, and grid energy storage.

The battery management system (BMS) is one of the most important parts of an electric vehicle [2]. State of charge (SOC) represents the remaining charge of the battery and is an important assessment of the battery state. The SOC cannot be directly measured. Therefore, the estimation of the SOC is not only an important function of the BMS, but is also a fundamental research topic in terms of BMSs. SOC estimation algorithms can be divided into two categories: model-based and non-model-based. Model-based algorithms have better performance in general. Some battery model examples include the Thevenin model, the Partnership for a New Generation of Vehicles (PNGV) model, the general nonlinear (GNL) model, the Rint model, and so on [3]. The majority of existing model-based SOC estimation algorithms use fixed model parameters, which are obtained by offline identification of battery test data. Many model-based algorithms are proposed for SOC estimation, such as the 
nonlinear Kalman filter [4-9], particle filtering (PF) [10,11], sliding mode observer (SMO) [12,13], the $H_{\infty}$ filter [14,15], and so on [16]. However, during the operation of an electric vehicle, factors such as temperature, SOC, and battery aging affect the battery model parameters, resulting in an increase in the SOC estimation error. Parameter identification is an important function of the BMS. Accurate model parameters can improve the estimation accuracy for model-based SOC estimation algorithms $[17,18]$. Constantly updating the parameters of the battery model, also known as online parameter identification, can effectively solve this problem for BMSs. Online parameter identification is a system identification problem, and research methods include the least-squares method [19-21], Lyapunov's direct method [22], the Kalman filter [23], and so on [24,25].

This paper proposes an online parameter identification algorithm and applies it to SOC estimation. During the SOC estimation process, the model parameters are continuously updated to reduce the SOC estimation error. As a result, the algorithm proposed in the paper provides a way to improve the existing production vehicles and other production battery-pack systems for industrial applications.

In a previous work [26], the battery was modeled by the Thevenin model, and the online parameter identification of the battery was realized by forgetting factor recursive least squares (FFRLS). A joint algorithm based on FFRLS and the unscented Kalman filter (UKF) (FFRLS-UKF) that estimates the SOC with model parameters constantly updating was proposed, and the methods were verified by experiments. In the above work, the forgetting factor was a fixed value. At different stages of the battery operation, according to the characteristics of the system, there are different requirements for forgetting factors. As a result, a variable forgetting factor (VFF) strategy is possible for improving the performance of FFRLS.

Many ways to adjust forgetting factors have been proposed in the literature. The Gauss-Newton variable forgetting factor recursive least squares (GN-VFF-RLS) algorithm uses the second derivatives of the cost function as the increase in the forgetting factor. GN-VFF-RLS has a higher tracking capability for parameter estimation [27]. Gradient-based variable forgetting factor recursive least squares (GVFF-RLS) uses a gradient-based method to modify the forgetting factor [28]. The gradient is derived from an improved mean-square-error analysis of recursive least squares. For an unknown system, the output is corrupted by a noise-like signal. This signal should be recovered in the filter. On the basis of this condition, another method for the variable forgetting factor recursive least squares (VFF-RLS) algorithm was designed for parameter identification [29]. For impulsive noises, a novel recursive logarithmic least-mean $p$ th (RLLMP) algorithm can enhance the tracking performance in the Volterra system [30]. On the basis of local polynomial modeling of the unknown time-varying (TV) system, a novel diffusion variable forgetting factor recursive least squares (Diff-VFF-RLS) algorithm was proposed [31]. Chen proposed a VFF algorithm using the exponential function [32]. Another method to change the forgetting factor is based on the curve of the inverse cotangent function [33].

This paper analyzes the possible ways to improve recursive least squares with a fixed forgetting factor. A VFF strategy is added to improve the FFRLS. A novel method for online parameter identification is proposed for lithium-ion batteries. Combined with the UKF, the VFF-RLS-UKF algorithm for SOC estimation is proposed. A series of experiments verified that the VFF strategy can improve the identification stability. The comparison with the measured value shows that the VFF-RLS-UKF algorithm can accurately estimate the battery SOC and terminal voltage.

This paper is arranged as follows: Section 2 introduces the FFRLS and analyzes the characteristics of forgetting factors. The VFF strategy is introduced to adapt to the requirements of the system, forming the VFF-RLS algorithm. In Section 3, a lithium-ion battery is modeled using the Thevenin model. An online parameter identification method based on the VFF-RLS algorithm is proposed. Combined with UKF, the VFF-RLS-UKF algorithm is proposed for SOC estimation. In Section 4, the experiments are introduced. The results of the online parameter identification by FFRLS and VFF-RLS are shown. The SOC and terminal voltage were estimated by the UKF, FFRLS-UKF, and VFF-RLS-UKF. In Section 5 , the conclusions are summarized. 


\section{Method and Analysis of Variable Forgetting Factor Strategy for Recursive Least Squares}

\subsection{Features of Forgetting Factor Recursive Least Squares}

As a classic approach for system identification, the recursive least squares (RLS) algorithm identifies the parameters of the system model by minimizing the sum of squares of the generalized errors. On the basis of RLS, the FFRLS algorithm was developed for systems with time-varying parameters. The process of FFRLS is briefly presented below [34].

A single-input, single-output system is described as

$$
A\left(z^{-1}\right) y(k)=z^{-d} B\left(z^{-1}\right) u(k)+\xi(k)
$$

where $u(k)$ is the input, $y(k)$ is the output, $\xi(k)$ is the white noise, and $z$ is the unit delay operator; $n_{a}, n_{b}$, and $d$ are known, and

$$
\left\{\begin{array}{l}
A\left(z^{-1}\right)=1+a_{1} z^{-1}+a_{2} z^{-2}+\cdots+a_{n_{a}} z^{-n_{a}} \\
B\left(z^{-1}\right)=b_{0}+b_{1} z^{-1}+b_{2} z^{-2}+\cdots+b_{n_{b}} z^{-n_{b}}
\end{array}\right.
$$

On the basis of the measurable data of the input and output, the following $\left(n_{a}+n_{b}+1\right)$ parameters are obtained by FFRLS:

$$
a_{1}, a_{2}, \cdots, a_{n_{a}} ; b_{0}, b_{1}, b_{2}, \cdots, b_{n_{b}} .
$$

Equation (1) can be converted to

$y(k)=-a_{1} y(k-1)-\cdots-a_{n_{a}} y\left(k-n_{a}\right)+b_{0} u(k-d)+\cdots+b_{n_{b}} u\left(k-d-n_{a}\right)+\xi(k)=\varphi^{T}(k) \theta+\xi(k)$,

where data vector $\varphi(k)$ and parameter vector $\theta$ are

$$
\left\{\begin{array}{l}
\varphi(k)=\left[-y(k-1), \cdots,-y\left(k-n_{a}\right), u(k-d), \cdots, u\left(k-d-n_{a}\right)\right]^{T} \in R^{\left(n_{a}+n_{b}+1\right) \times 1} \\
\theta=\left[a_{1}, \cdots, a_{n_{a}}, b_{0}, \cdots, b_{n_{b}}\right]^{T} \in R^{\left(n_{a}+n_{b}+1\right) \times 1}
\end{array}\right.
$$

The cumulative squared error can be described as

$$
J_{0}=\sum_{k=1}^{L} \lambda^{L-k}\left[y(k)-\varphi^{T}(k) \hat{\theta}\right]^{2}
$$

where $L$ is the number of observations and $\lambda$ is the forgetting factor. In order to minimize the cumulative squared error, recursive formulas are deduced as

$$
\left\{\begin{array}{l}
\hat{\theta}(k)=\hat{\theta}(k-1)+K(k)\left[y(k)-\varphi^{T}(k) \hat{\theta}(k-1)\right] \\
K(k)=\frac{P(k-1) \theta(k)}{\lambda+\varphi^{T}(k) P(k-1) \varphi(k)} \\
P(k)=\frac{1}{\lambda}\left[I-K(k) \varphi^{T}(k)\right] P(k-1)
\end{array}\right.
$$

The value of the forgetting factor $\lambda$ has a significant effect on the performance of the system identification. According to Equation (5), $J_{0}$ is the weighted sum of squared errors at different times. The weight of the $L$ th observation is 1 , while the weight of the $(L-n)$ th observation is $\lambda^{n}$. As time passes, the impact of early data gradually diminishes. The value of the forgetting factor affects the rate of data weight attenuation. When the forgetting factor goes to lower values, the latest data has a significant impact on $J_{0}$. That is to say, FFRLS can track the changes of the parameters quickly. However, at the same time, the stability of the algorithm is reduced, and it is easy for it to diverge. When the forgetting factor approaches 1, the stability of FFRLS is high, but the ability to track time-varying parameters is weak. We note that, when the forgetting factor is equal to 1 , the algorithm degenerates into ordinary RLS. 


\subsection{Variable Forgetting Factor Strategy Considering Errors}

In previous work [26], FFRLS was used to identify the parameters of the Thevenin model online. During battery operation, FFRLS-UKF estimates the SOC with model parameters constantly updated. In the above work, the forgetting factor was a fixed value. However, at different moments of the battery operation, there are different requirements for forgetting factors [29]. Therefore, a VFF strategy is possible for improving the performance of online parameter identification and SOC estimation.

As shown in Equation (7), the error of FFRLS is defined as the difference between the output observation and the predicted value:

$$
e(k)=y(k)-\varphi^{T}(k) \hat{\theta}
$$

The stability degree of the algorithm can be indicated by the error. In practical applications, the parameter change does not maintain a certain predictable trend, and the error in the calculation process will change with time. Therefore, RLS with a fixed forgetting factor can be improved by adjusting the forgetting factor constantly. The forgetting factor, if adjusted according to the change in the error at different moments, will possibly improve the performance. When the error is large, the algorithm tends to be unstable, and the parameters may have obvious changes. At this time, the forgetting factor should be properly reduced so that the performance of parameter tracking can be improved. When the error is small, this indicates that the current parameter identification result is close to the real value. At this time, no major modification of the parameters is needed, but the stability of the algorithm needs to be improved. Therefore, the forgetting factor should be increased appropriately. On the basis of the above analysis and the idea of the VFF designs in Section 1, a VFF strategy is introduced as follows.

On the basis of the VFF strategy, methods of online parameter identification and SOC estimation were developed and verified by experiments, which is relevant given the minor improvements to state-of-the art methods.

The VFF can be derived as

$$
\left\{\begin{array}{l}
\lambda(k)=\lambda_{\min }+\left(1-\lambda_{\min }\right)^{\alpha(k)} \\
\alpha(k)=2^{\rho e^{2}(k)}
\end{array}\right.
$$

where $\lambda_{\min }$ and $\rho$ are fixed parameters.

Considering that $e(k)$ can be either positive or negative, $e^{2}(k)$ is used to describe the error. It can be seen from the Equation (7) that when $e^{2}(k)$ approaches $0, \alpha(k)$ and $\lambda(k)$ approach 1 ; when $e^{2}(k)$ approaches infinity, $\alpha(k)$ approaches infinity and $\lambda(k)$ approaches $\lambda_{\text {min }}$.

Setting $\lambda_{\min }=0.8$ and $\rho=10,000$, we observe that $\lambda(k)$ changes with $e(k)$. In Figure 1 , the solid blue line indicates $\lambda(k)$, and the two red broken lines indicate the maximum value of 1 and the minimum value of 0.8 for $\lambda(k)$.

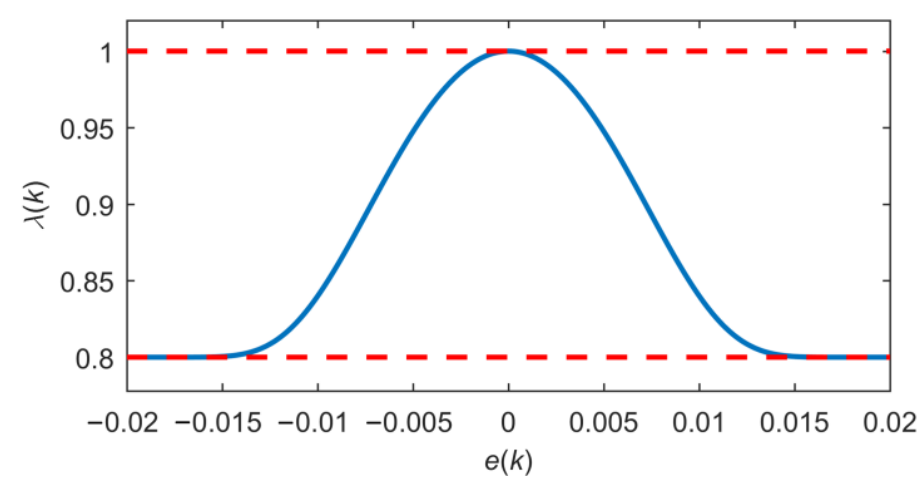

Figure 1. Forgetting factor $\lambda(k)$ curve with error $e(k)$. 
It can be seen that when $e^{2}(k)=0$ and $\lambda(k)=1$, when $e^{2}(k)$ increases, $\lambda(k)$ gradually decreases; when $e^{2}(k)$ approaches infinity, $\lambda(k)$ approaches $\lambda_{\min }=0.8$. The strategy to adjust the forgetting factor is in line with expectations. We note that in this case, the values of $\lambda_{\min }$ and $\rho$ are arbitrary, and the values do not affect the shape of the curve.

\subsection{Effect of Strategy Parameters on Variable Forgetting Factor}

The curve of the forgetting factor $\lambda(k)$ as a function of the error $e(k)$ is affected by the strategy parameters $\lambda_{\min }$ and $\rho ; \lambda_{\min }$ determines the minimum value of $\lambda(k)$. For any $e(k), \lambda_{\min } \leq \lambda(k) \leq 1$. The parameter $\rho$ adjusts the sensitivity of the forgetting factor to the error. When $\rho$ is set to a large value, $\lambda(k)$ is sensitive to $e(k)$, and a slight increase in $e(k)$ can reduce $\lambda(k)$ significantly. When $\rho$ is set to a small value, $e(k)$ needs to be larger to obtain a small $\lambda(k)$.

We define the judging indicator as

$$
J=\sum_{k=1}^{L}\left[y(k)-\varphi^{T}(k) \hat{\theta}\right]^{2}
$$

As the sum of squared errors at different times with equal weights, $J$ can be used to evaluate whether the values of $\lambda_{\min }$ and $\rho$ are appropriate.

\section{Novel Methods for Lithium-Ion Battery Online Parameter Identification and State of Charge Estimation}

\subsection{Battery Modeling}

The Thevenin model is used as the equivalent circuit model for a lithium-ion battery. As shown in Figure 2, the Thevenin model is made up of a voltage source $u_{\mathrm{oc}}$, ohmic resistance $R_{0}$, a parallel link of polarization resistor $R_{\mathrm{p}}$, and a polarization capacitor $C_{\mathrm{p}} ; i$ and $u_{\mathrm{t}}$ the indicate current and terminal voltages, respectively, and $u_{\mathrm{p}}$ is the voltage of the resistor-capacitor (RC) link.

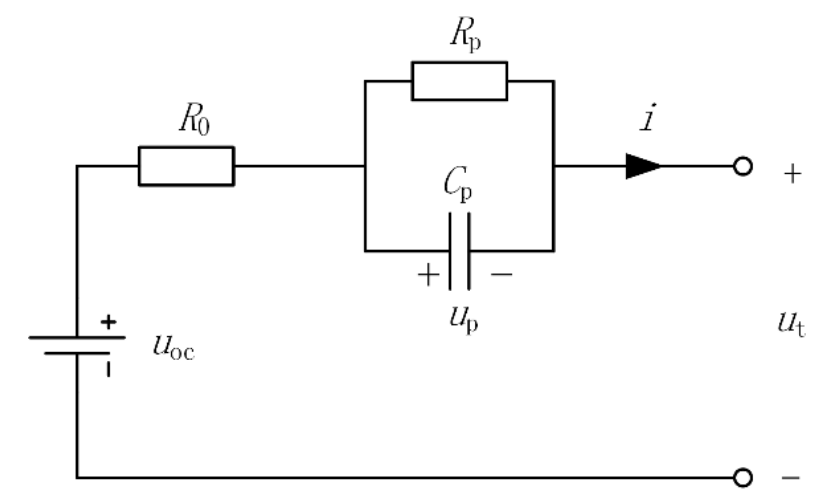

Figure 2. Thevenin model.

According to Kichhoff's law, $u_{\mathrm{oc}}$ can be expressed as:

$$
u_{\mathrm{oc}}=u_{\mathrm{t}}+i R_{0}+u_{\mathrm{p}}
$$

Based on the relationship between the current and voltage of $C_{\mathrm{p}}$, we can derive:

$$
C_{\mathrm{p}} \frac{d u_{\mathrm{p}}}{d t}+\frac{u_{\mathrm{p}}}{R_{\mathrm{p}}}=i
$$

The value of the voltage source is written as $u_{\mathrm{oc}}$ because the voltage of the voltage source is equal to the open-circuit voltage (OCV). When a battery is left unpowered for a long enough period of 
time, the terminal voltage tends to be a certain stable value, known as the OCV. There is a one-to-one correspondence between the $\mathrm{OCV}$ and $\mathrm{SOC}$, and the OCV-SOC curve is one of the basic characteristics of a battery.

\subsection{Method for Online Parameter Identification on the Basis of Variable Forgetting Factor Recursive} Least Squares

There are several definitions of state of health $(\mathrm{SOH})$. In this paper, the ohmic resistance $R_{0}$ is used to evaluate the $\mathrm{SOH}$ of lithium-ion batteries. The results of online parameter identification can also be used to evaluate the battery's $\mathrm{SOH}$.

Equations (10) and (11) are processed by the Laplace transform and discretization as

$$
\left(\frac{R_{\mathrm{p}} C_{\mathrm{p}}}{T}+1\right)\left(u_{\mathrm{t}}-u_{\mathrm{oc}}\right)(k)=\frac{R_{\mathrm{p}} C_{\mathrm{p}}}{T}\left(u_{\mathrm{t}}-u_{\mathrm{oc}}\right)(k-1)-\left(\frac{R_{0} R_{\mathrm{p}} C_{\mathrm{p}}}{T}+R_{0}+R_{\mathrm{p}}\right) i(k)+\frac{R_{0} R_{\mathrm{p}} C_{\mathrm{p}}}{T} i(k-1),
$$

where $T$ is the sampling period. Equation (12) can be converted to the simplest form:

$$
\left(u_{\mathrm{t}}-u_{\mathrm{oc}}\right)(k)=-k_{1}\left(u_{\mathrm{t}}-u_{\mathrm{oc}}\right)(k-1)+k_{2} i(k)+k_{3} i(k-1)
$$

The format of Equation (13) is the same that of as Equation (3). The current $i$ is set as the input, and the voltage difference $\left(u_{\mathrm{t}}-u_{\mathrm{oc}}\right)$ is set as the output; $k_{1}, k_{2}$, and $k_{3}$ can be identified by VFF-RLS, and $R_{0}, R_{\mathrm{p}}$, and $C_{\mathrm{p}}$ can be derived as follows:

$$
\left\{\begin{array}{l}
R_{0}=-\frac{k_{3}}{k_{1}} \\
R_{\mathrm{p}}=-\frac{k_{2}+R_{0}}{k_{1}+1} \\
C_{\mathrm{p}}=\frac{\left(\frac{1}{k_{1}+1}-1\right) T}{R_{\mathrm{p}}}
\end{array}\right.
$$

It can be seen that the $\mathrm{SOH}$ (ohmic resistance) can also be estimated by the VFF-RLS algorithm.

\subsection{Joint Algorithm of State of Charge Estimation}

The definition of SOC is

$$
\operatorname{SOC}(t)=\operatorname{SOC}\left(t_{0}\right)-\frac{\int_{t_{0}}^{t} i \mathrm{~d} t}{C_{\mathrm{n}}}
$$

where $C_{\mathrm{n}}$ denotes the nominal capacity of the battery.

The UKF is a nonlinear Kalman filter algorithm that is suitable for strong nonlinear systems. The process equation of a lithium battery can be derived from Equations (11) and (15) as

$$
x(k)=\left(\begin{array}{cc}
1 & 0 \\
0 & 1-\frac{T}{C_{\mathrm{p}} R_{\mathrm{p}}}
\end{array}\right) x(k-1)+\left(\begin{array}{c}
-\frac{T}{C_{\mathrm{p}}} \\
\frac{T}{C_{\mathrm{p}}}
\end{array}\right) i(k)
$$

where $x(k)$ is the state vector:

$$
x(k)=\left(\begin{array}{c}
\mathrm{SOC}(k) \\
u_{\mathrm{p}}(k)
\end{array}\right) .
$$

The measurement equation of the UKF can be derived from Equations (10) and (15) as

$$
u_{\mathrm{t}}(k)=u_{\mathrm{oc}}(k)-i(k) R_{0}-u_{\mathrm{p}}(k),
$$

where $u_{\mathrm{t}}(k)$ is the measurement vector and $u_{\mathrm{oc}}(k)=f[\operatorname{SOC}(k)]$ is a nonlinear function. According to the process equation and the measurement equation, the state vector can be estimated by the UKF, and the SOC can be obtained.

A joint SOC estimation method based on VFF-RLS and the UKF with online parameter identification is proposed. Figure 3 illustrates the schematic of the VFF-RLS-UKF algorithm. 


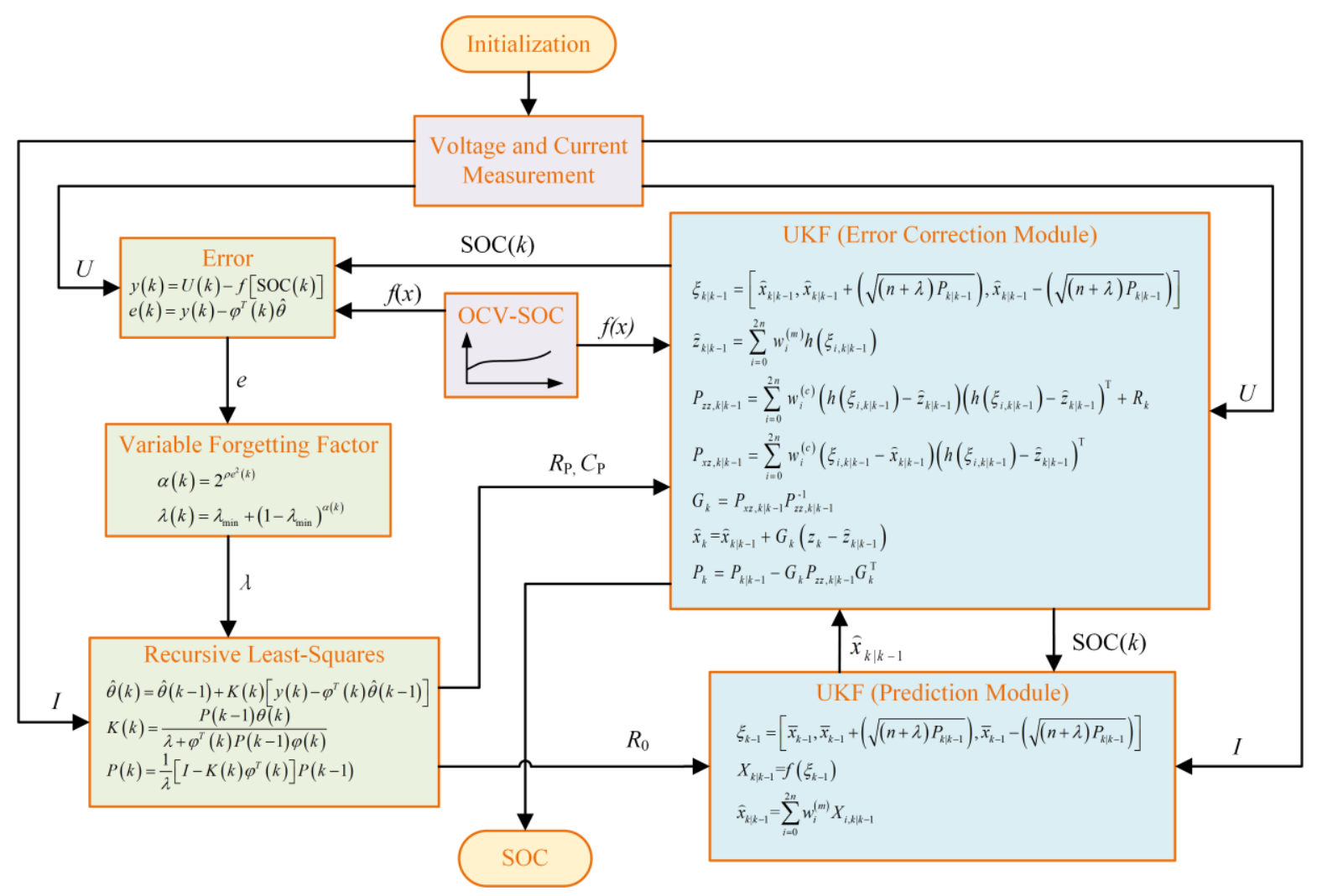

Figure 3. Schematic of variable forgetting factor recursive least squares unscented Kalman filter (VFF-RLS-UKF) algorithm.

The algorithm is explained as follows: First, initialize all the variables used in the algorithm. In each step of the loop operation, it is necessary to measure the battery operating current and terminal voltage. Calculate the error on the basis of the measured terminal voltage, the last SOC estimate, and the OCV-SOC curve. Next, the new forgetting factor is calculated, and the battery model parameters are updated. According to the battery model parameters and measured values, the current SOC is estimated and output. Then, the algorithm goes to the next cycle. We note that the OCV-SOC curve was measured experimentally.

\section{Experiment and Discussion}

\subsection{Capacity Test and OCV-SOC Curve Test of Lithium-Ion Battery}

In general, there are three types of packages of lithium-ion batteries: cylindrical, pouch, and prismatic [35]. These have similar electrochemical principles and charge-discharge characteristics. The technology for producing cylindrical batteries is the earliest and most mature. The 18650 battery, a typical cylindrical battery, has reached a very high level of consistency and safety, although its capacity is relatively small. Many battery packs in electric vehicles are made up of 18650 batteries, such as the Tesla Model S. In experiments, the Samsung ICR18650-22P battery was chosen as the experimental object, which was representative of the research into lithium-ion batteries of electric vehicles.

Figure 4 illustrates the configuration of the battery test bench. In the experiment, the charge and discharge program was designed on a personal computer (PC). The subject in the experiment was lithium-ion batteries (ICR18650-22P, Samsung, Seoul, South Korea). A battery testing system (BT-5HC, Arbin, College Station, TX, USA) received instructions from the PC to charge and discharge the battery. The voltage, current and temperature data were measured by the battery testing system 
and transported to the PC. During the experiment, a temperature chamber (SC-80-CC-2, Sanwood, Dongguan, China) provided the battery with the desired working temperature.

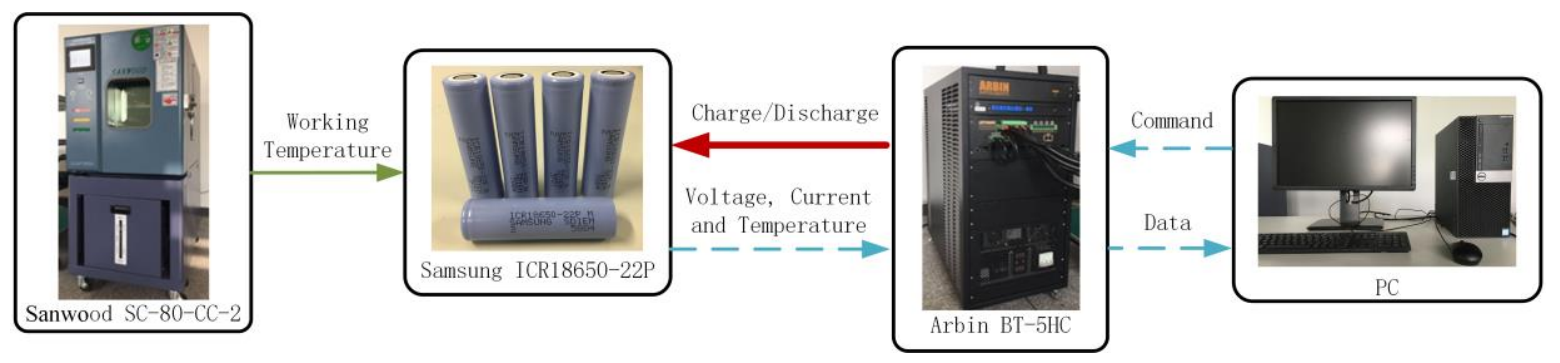

Figure 4. Test bench configuration.

As the basis for other experiments, the nominal capacity of the battery was measured in the experiment. The temperature was set to $25^{\circ} \mathrm{C}$, and the battery was discharged from full to no charge. The amount of electricity discharged during the process gave the capacity of the battery. After three repetitions, the average value was obtained as the measured capacity of the battery. The battery capacity in the experiment was $2.039 \mathrm{Ah}$.

As stated in Section 3.1, the OCV-SOC curve is one of the basic characteristics of a battery and is essential in online parameter identification and SOC estimation. The experimental temperature was $25^{\circ} \mathrm{C}$. In the case of multiple SOCs (13 SOCs in this experiment), after long enough periods of rest, the OCVs were recorded and plotted in the coordinate system. Polynomial fitting was performed on the 13 measured points to obtain the functional relationship between the OCV and SOC. The curve is shown in Figure 5, and the function is

$$
\mathrm{OCV}=14.461 \cdot \mathrm{SOC}^{6}-36.156 \cdot \mathrm{SOC}^{5}+30.283 \cdot \mathrm{SOC}^{4}-8.660 \cdot \mathrm{SOC}^{3}-0.044 \cdot \mathrm{SOC}^{2}+0.861 \cdot \mathrm{SOC}+3.4453
$$

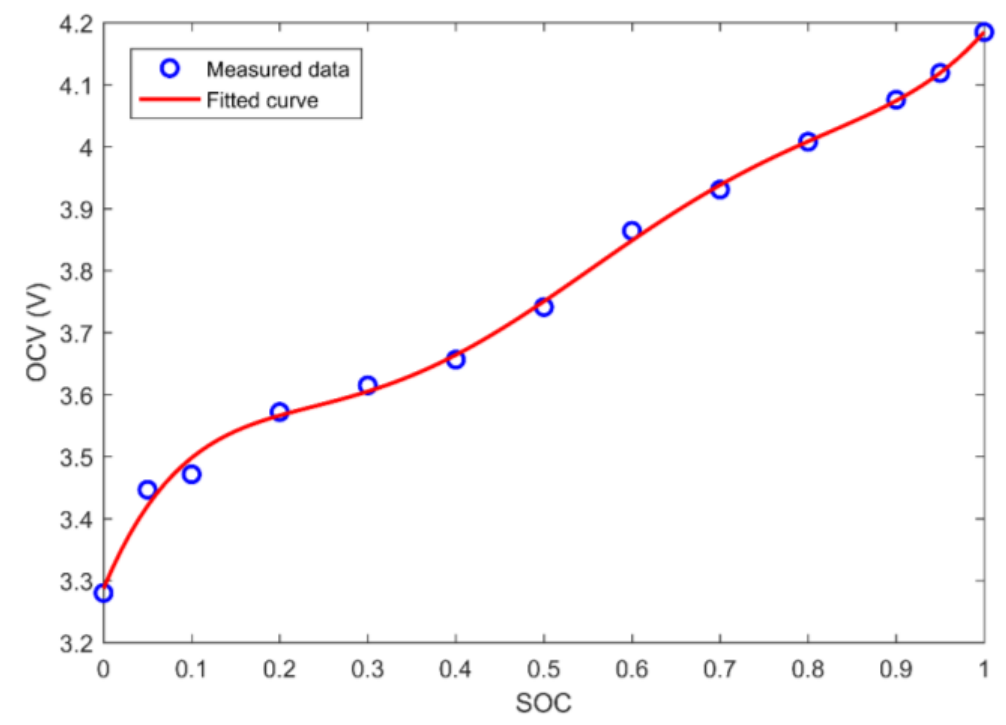

Figure 5. Measured points and fitted open-circuit voltage-state of charge (OCV-SOC) curve at $25^{\circ} \mathrm{C}$.

\subsection{Results of Online Parameter Identification by FFRLS and VFF-RLS}

At $25^{\circ} \mathrm{C}$, the New European Driving Cycle (NEDC) was loaded on the battery to simulate the working process of the battery in an electric vehicle. Gaussian white noise was added to the original data to simulate the real situation. In Section 3.2, Equation (13) is the battery model in the least-squares 
form. The FFRLS and VFF-RLS algorithms could be used to identify the model parameters $R_{0}, R_{\mathrm{p}}$, and $C_{\mathrm{p}}$ online.

For FFRLS, the value of the fixed forgetting factor $\lambda$ affected the identification results. After several tests, it was found that when $\lambda=0.97$, the judging indicator from Equation (9) reached the minimum value of $J=0.0413$. At this point, the overall error could be considered to be minimal. Similarly, for VFF-RLS, when selecting a different $\left(\lambda_{\min }, \rho\right)$ set, the values of $J$ were as shown in Figure 6 . It can be seen that the surface was continuous and the optimal $\left(\lambda_{\min }, \rho\right)$ set was unique for $J$ to reach the minimum. When $\lambda_{\min }=0.75$ and $\rho=33000, J$ reached the minimum at $J=0.0390$. It can be seen that VFF-RLS could make the overall error of the system smaller compared with FFRLS.

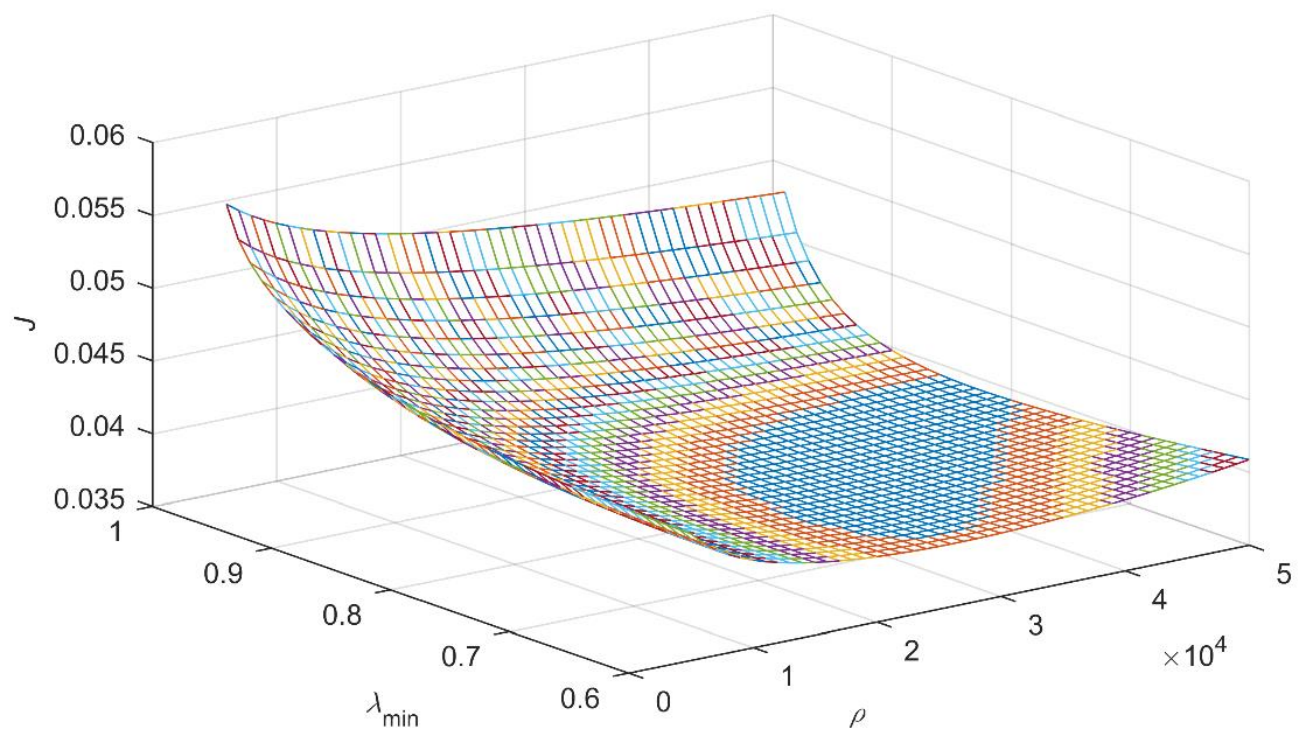

Figure 6. Judging indicator by variable forgetting factor recursive least squares (VFF-RLS) with different $\left(\lambda_{\min }, \rho\right)$ sets.

According to the shape of the surface, it can be seen that the surface was flat near the optimal $\left(\lambda_{\min }, \rho\right)$ set, which means that if the $\left(\lambda_{\min }, \rho\right)$ set changed within a certain range, $J$ did not change greatly. That is to say, the VFF-RLS algorithm can achieve good results when the $\left(\lambda_{\min }, \rho\right)$ values are set within a certain range.

When $\lambda_{\min }=0.75$ and $\rho=33,000$, the curve of the forgetting factor versus time and the curve of the error versus time were as shown in Figure 7 . The forgetting factor and error at all times of the NEDC test were as shown in Figure 7a,b. Figure 7c,d shows parts of Figure 7a,b, respectively. It can be seen that when the absolute value of the error was large, the value of the forgetting factor was relatively small; when the absolute value of the error was small, the value of the forgetting factor was close to 1 . This was consistent with the theory.

The results of the online parameter identification are shown in Figure 8. It is shown that both methods converged quickly and achieved stable values. The results of the VFF-RLS algorithm fluctuated less compared to the FFRLS algorithm. The reference values in the figure were identified by the offline method [36]. In general, the ohmic resistance identified offline in a constant temperature environment was considered quite accurate. However, other results of offline identification were considered to have considerable errors. 

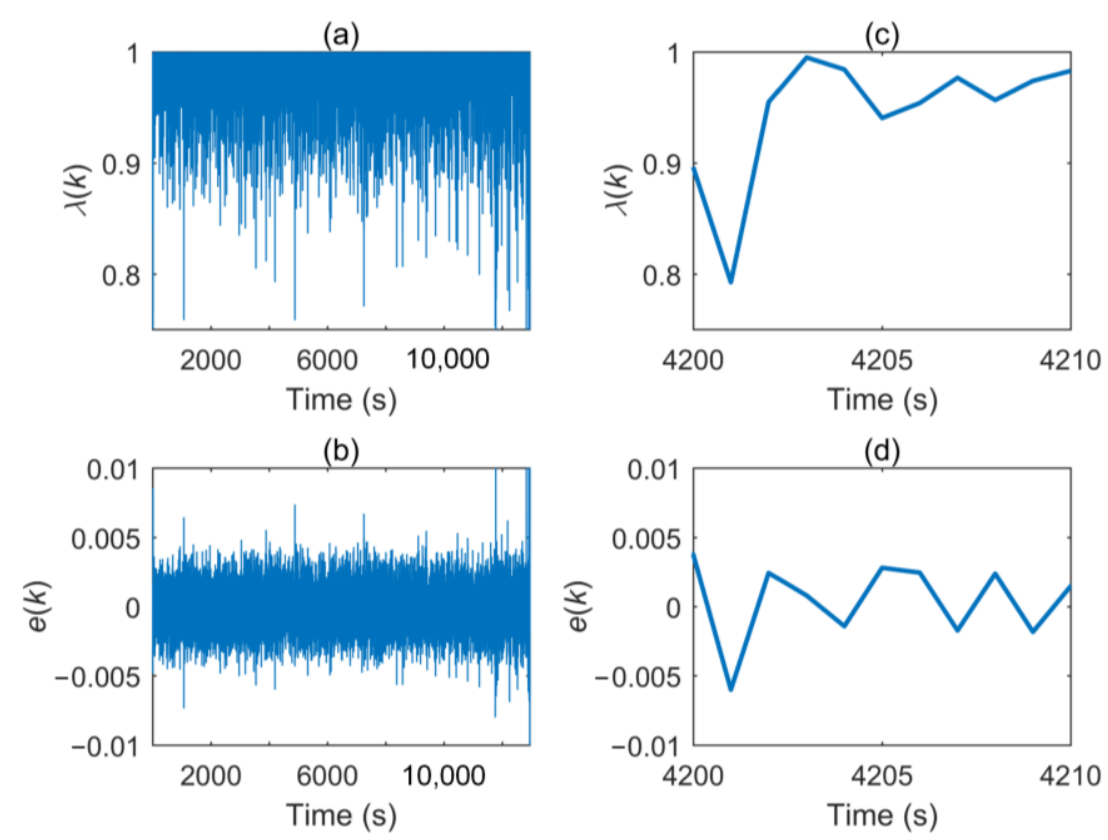

Figure 7. Forgetting factor and error over time in New European Driving Cycle (NEDC) test: (a) forgetting factor at all times; (b) error at all times; (c) forgetting factor from 4200 to $4210 \mathrm{~s}$; (d) error from 4200 to $4210 \mathrm{~s}$.
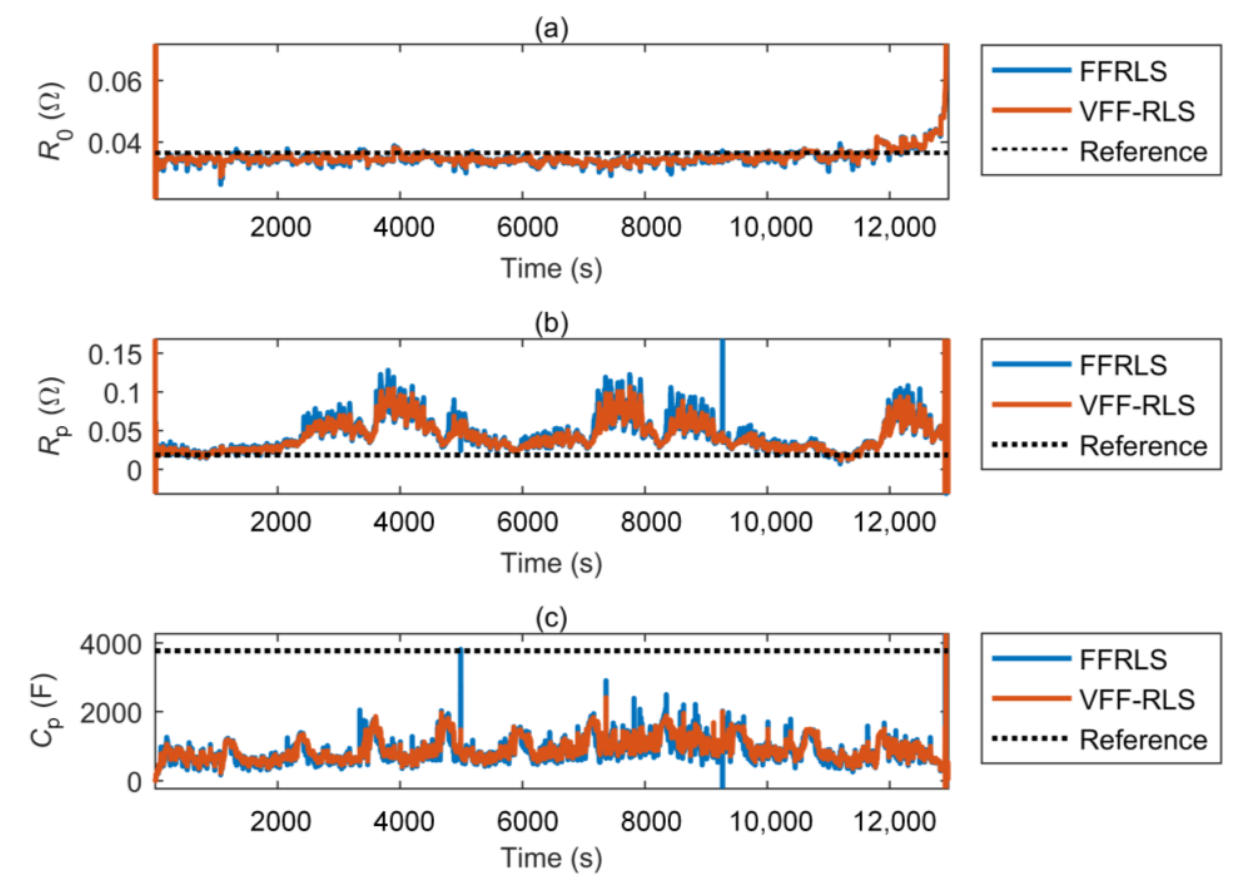

Figure 8. Results of online parameter identification: (a) ohmic resistance $R_{0}$; (b) polarization resistor $R_{\mathrm{p}} ;(\mathbf{c})$ polarization capacitor $C_{\mathrm{p}}$.

\subsection{Results of SOC and Terminal Voltage Estimation by UKF, FFRLS-UKF, and VFF-RLS-UKF}

In the SOC estimation experiment, the battery was loaded with the NEDC current under variable-temperature ambient conditions. The temperature ranged from 5 to $45{ }^{\circ} \mathrm{C}$, imitating the actual working environment of an electric vehicle. The NEDC is a driving cycle that is designed to assess passenger cars. It was simulated to obtain current data in ADVISOR $[37,38]$. Considering the 
experimental battery the loaded current in the experiment was scaled to a maximum current of $5 \mathrm{~A}$. The purpose of the experimental setup was to simulate the operation of batteries in an electric vehicle.

Because the battery parameters are mainly affected by the temperature, SOC, and aging degree, the temperature and SOC changed significantly during the experiment, causing significant changes in the model parameters. As a result, the experiment was representative to test the ability of the SOC estimation algorithm to overcome the effect of parameter changes.

The current, voltage, and temperature of the SOC estimation experiment are shown in Figure 9.

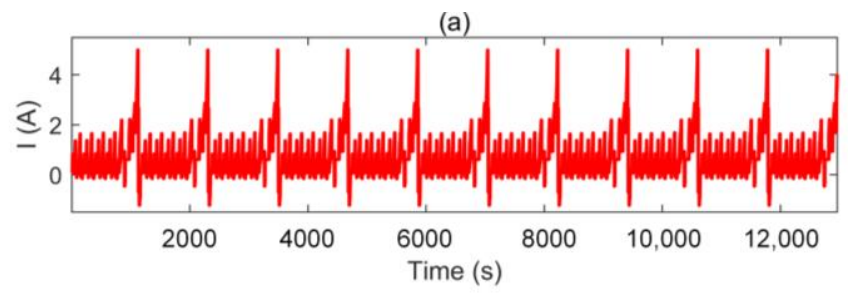

(b)

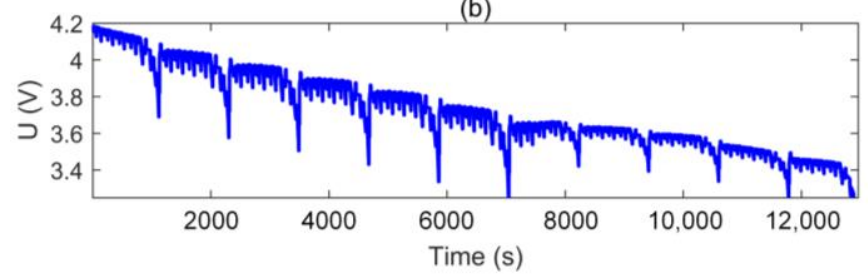

(c)

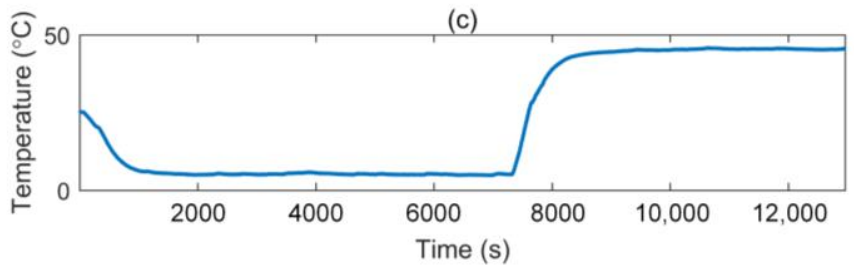

Figure 9. Results of New European Driving Cycle (NEDC) test in variable-temperature environment:

(a) current; (b) terminal voltage; (c) temperature.

On the basis of the battery test data, the SOC was estimated separately by the UKF, FFRLS-UKF, and VFF-RLS-UKF. The results of the SOC estimation are listed in Table 1 and shown in Figure 10. The reference value of the SOC was obtained by the ampere-hour integral method, as this measurement is very accurate.

During the UKF operation, the model parameters were regarded as constant. As a result, the SOC estimation error by the UKF had the largest average and maximum values among the three methods. The average errors of FFRLS-UKF or VFF-RLS-UKF were less than $1 \%$, indicating that they effectively tracked the changes of the battery model parameters. Compared with FFRLS-UKF, the SOC estimate of VFF-RLS-UKF was more accurate, indicating that the VFF improved the performance of RLS. The root-mean-square error (RMSE) assesses the stability of estimation results. The result of VFF-RLS-UKF was the most stable of the three methods.

Table 1. Results of state of charge (SOC) estimation.

\begin{tabular}{cccc}
\hline Method & UKF $^{\mathbf{1}}$ & FFRLS-UKF $^{2}$ & VFF-RLS-UKF $^{3}$ \\
\hline Mean error & 0.04398 & 0.00926 & 0.00595 \\
Max error & 0.06001 & 0.01391 & 0.00871 \\
RMSE $^{4}$ & 0.04767 & 0.00989 & 0.00630 \\
\hline
\end{tabular}

${ }^{1}$ unscented Kalman filter (UKF) ${ }^{2}$ forgetting factor recursive least squares unscented Kalman filter (FFRLS-UKF)

${ }^{3}$ variable forgetting factor recursive least squares unscented Kalman filter (VFF-RLS-UKF) ${ }^{4}$ root-mean-square error (RMSE). 
(a)

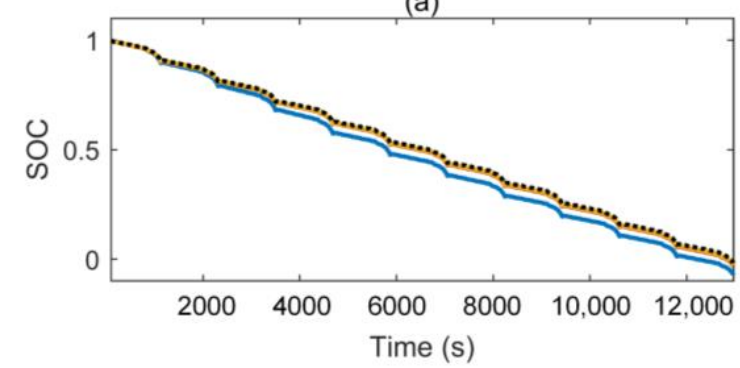

(b)

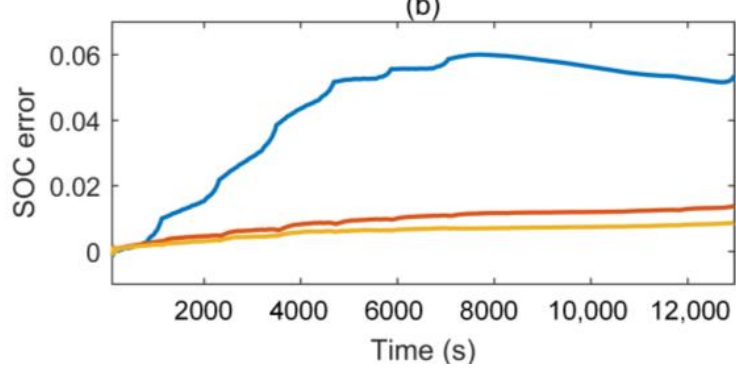

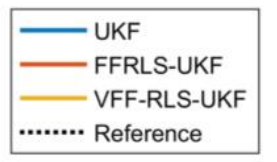

UKF

FFRLS-UKF

VFF-RLS-UKF

Figure 10. Results of state of charge (SOC) estimation by unscented Kalman filter (UKF), forgetting factor recursive least squares-UKF (FFRLS-UKF), and variable forgetting factor RLS-UKF (VFF-RLS-UKF): (a) SOC estimation; (b) SOC estimation error.

Because the terminal voltage is a measurement vector in the UKF, it is constantly being estimated during the operation of the algorithm. The performances of the three methods can be visually compared in terms of the estimated and measured values of the terminal voltage. The results of the terminal voltage estimation are listed in Table 2 and shown in Figure 11.

It can be seen that all three algorithms could correctly estimate the terminal voltage. Regarding the average of the error, the maximum value of the error, and the RMSE, shown in Table 2, the UKF had the worst effect, and VFF-RLS-UKF was slightly better than FFRLS-UKF.

(a)

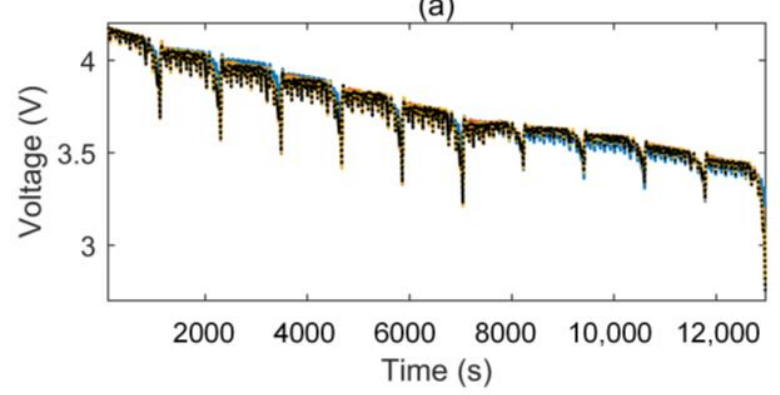

(b)

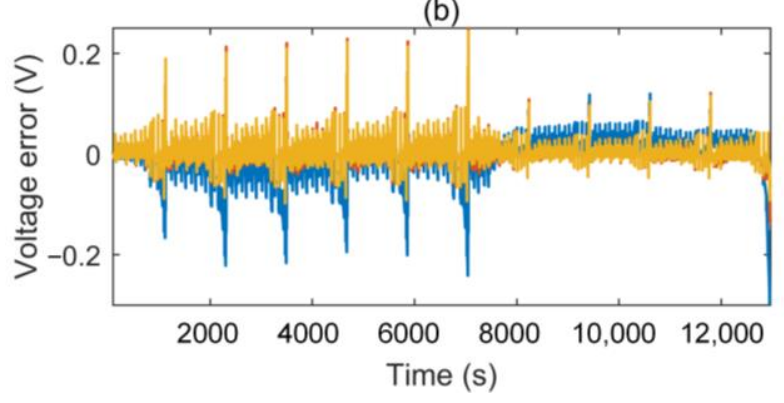

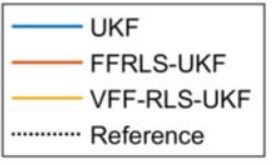

UKF

FFRLS-UKF

VFF-RLS-UKF

Figure 11. Results of terminal voltage estimation by unscented Kalman filter (UKF), forgetting factor recursive least squares-UKF (FFRLS-UKF), and variable forgetting factor RLS-UKF (VFF-RLS-UKF):

(a) terminal voltage estimation; (b) terminal voltage estimation error. 
Table 2. Results of terminal voltage estimation.

\begin{tabular}{cccc}
\hline Method & UKF & FFRLS-UKF & VFF-RLS-UKF \\
\hline Mean error & 0.02696 & 0.00843 & 0.00687 \\
Max error & 0.45917 & 0.24947 & 0.24345 \\
RMSE & 0.04112 & 0.01393 & 0.01224 \\
\hline
\end{tabular}

\section{Conclusions}

A VFF strategy is introduced in this paper to automatically adjust the forgetting factor and improve the performance of RLS. A judging indicator that represents the overall system error is proposed as a reference for parameter selection. The lithium-ion battery is modeled by the Thevenin model. The online identification method of the battery model parameters is proposed on the basis of the VFF-RLS algorithm. A battery was tested with the NEDC at a constant temperature of $25^{\circ} \mathrm{C}$. The FFRLS and VFF-RLS algorithms were used to identify the model parameters of the battery online. It could be seen that the model parameters identified by VFF-RLS became stable quickly, and the ohmic resistance was close to the offline measurement values. The results of VFF-RLS were more stable than those of the identification of FFRLS. In combination with the UKF, the VFF-RLS-UKF algorithm is proposed and can be used for SOC estimation. The SOC and terminal voltage can be used to verify the algorithm. The NEDC was used in an environment of variable temperature. The SOC and terminal voltage were estimated using the UKF, FFRLS-UKF, and VFF-RLS-UKF algorithms. For SOC estimation, the UKF estimation error that did not consider the parameter change problem was the largest, with an average error of $4.398 \%$. FFRLS-UKF was significantly more accurate, with an average error of $0.926 \%$. When the VFF-RLS-UKF algorithm set a proper strategy for the VFF, this further improved the accuracy, with an average error of $0.595 \%$. For terminal voltage estimation, the average errors of the UKF, FFRLS-UKF, and VFF-RLF-UKF were $2.696 \%, 0.843 \%$, and $0.687 \%$, respectively. The trend was the same as for the SOC estimation. The results show that VFF-RLS-UKF can accurately estimate the battery status and verify that the VFF strategy can improve the performance of RLS.

Author Contributions: Conceptualization: Z.L.; formal analysis: W.W. and W.S.; data curation: Y.L. and M.W.; writing, original draft: Z.L.; writing, review and editing: B.X.; supervision: B.X.

Acknowledgments: This work was supported by the Shenzhen Science and Technology Project (Grant No. JCY20150331151358137).

Conflicts of Interest: The authors declare no conflict of interest.

\section{Acronyms}

\begin{tabular}{ll}
\hline BMS & Battery management system \\
FFRLS & Forgetting factor recursive least squares \\
GNL & General nonlinear \\
NEDC & New European Driving Cycle \\
OCV & Open-circuit voltage \\
PC & Personal computer \\
PF & Particle filtering \\
PNGV & Partnership for a New Generation of Vehicles \\
RLS & Recursive least squares \\
RMSE & Root-mean-square error \\
SMO & Sliding mode observer \\
SOC & State of charge \\
SOH & State of health \\
UKF & Unscented Kalman filter \\
VFF & Variable forgetting factor \\
\hline
\end{tabular}




\section{References}

1. Blomgren, G.E. The development and future of lithium ion batteries. J. Electrochem. Soc. 2016, 164, A5019-A5025. [CrossRef]

2. Hoque, M.M.; Hannan, M.A.; Mohamed, A.; Ayob, A. Battery charge equalization controller in electric vehicle applications: A review. Renew. Sustain. Energy Rev. 2017, 75, 1363-1385. [CrossRef]

3. Zhang, X.; Zhang, W.; Lei, G. A review of li-ion battery equivalent circuit models. Trans. Electr. Electron. Mater. 2016, 17, 311-316. [CrossRef]

4. Plett, G.L. Extended Kalman filtering for battery management systems of LiPB-based HEV battery packs: Part 1. Background. J. Power Sources 2004, 134, 252-261. [CrossRef]

5. Plett, G.L. Extended Kalman filtering for battery management systems of LiPB-based HEV battery packs: Part 2. Modeling and identification. J. Power Sources 2004, 134, 262-276. [CrossRef]

6. Plett, G.L. Extended Kalman filtering for battery management systems of LiPB-based HEV battery packs: Part 3. State and parameter estimation. J. Power Sources 2004, 134, 277-292. [CrossRef]

7. He, Z.; Gao, M.; Wang, C.; Wang, L.; Liu, Y. Adaptive state of charge estimation for li-ion batteries based on an unscented Kalman filter with an enhanced battery model. Energies 2013, 6, 4134-4151. [CrossRef]

8. Sun, F.C.; Hu, X.S.; Zou, Y.; Li, S.G. Adaptive unscented Kalman filtering for state of charge estimation of a lithium-ion battery for electric vehicles. Energy 2011, 36, 3531-3540. [CrossRef]

9. Xia, B.; Wang, H.; Tian, Y.; Wang, M.; Sun, W.; Xu, Z. State of charge estimation of lithium-ion batteries using an adaptive cubature Kalman filter. Energies 2015, 8, 5916-5936. [CrossRef]

10. Xia, B.Z.; Sun, Z.; Zhang, R.F.; Lao, Z.Z. A cubature particle filter algorithm to estimate the state of the charge of lithium-ion batteries based on a second-order equivalent circuit model. Energies 2017, 10, 15. [CrossRef]

11. Xia, B.; Sun, Z.; Zhang, R.; Cui, D.; Lao, Z.; Wang, W.; Sun, W.; Lai, Y.; Wang, M. A comparative study of three improved algorithms based on particle filter algorithms in soc estimation of lithium ion batteries. Energies 2017, 10, 1149. [CrossRef]

12. Zhang, F.; Liu, G.; Fang, L. A battery state of charge estimation method using sliding mode observer. In Proceedings of the 7th World Congress on Intelligent Control and Automation, Chongqing, China, 25-27 June 2008; pp. 989-994.

13. Kim, I.S. The novel state of charge estimation method for lithium battery using sliding mode observer. J. Power Sources 2006, 163, 584-590. [CrossRef]

14. Yan, J.; Xu, G.; Xu, Y.; Xie, B. Battery state-of-charge estimation based on $H \infty$ filter for hybrid electric vehicle. In Proceedings of the 10th International Conference on Control, Automation, Robotics and Vision, Hanoi, Vietnam, 17-20 December 2008; pp. 464-469.

15. Zhang, F.; Liu, G.; Fang, L.; Wang, H. Estimation of battery state of charge with $H \infty$ observer: Applied to a robot for inspecting power transmission lines. IEEE Trans. Ind. Electron. 2012, 59, 1086-1095. [CrossRef]

16. Xia, B.; Zheng, W.; Zhang, R.; Lao, Z.; Sun, Z. A novel observer for lithium-ion battery state of charge estimation in electric vehicles based on a second-order equivalent circuit model. Energies 2017, 10, 1150. [CrossRef]

17. Cuma, M.U.; Koroglu, T. A comprehensive review on estimation strategies used in hybrid and battery electric vehicles. Renew. Sustain. Energy Rev. 2015, 42, 517-531. [CrossRef]

18. Xu, J.; Cao, B.; Cao, J.; Zou, Z. A comparison study of the model based soc estimation methods for lithium-ion batteries. In Proceedings of the 2013 IEEE Vehicle Power and Propulsion Conference (VPPC), Beijing, China, 15-18 October 2013; pp. 1-5.

19. Guo, X.; Kang, L.; Yao, Y.; Huang, Z.; Li, W. Joint estimation of the electric vehicle power battery state of charge based on the least squares method and the Kalman filter algorithm. Energies 2016, 9, 100. [CrossRef]

20. Duong, V.-H.; Bastawrous, H.A.; Lim, K.; See, K.W.; Zhang, P.; Dou, S.X. Online state of charge and model parameters estimation of the $\mathrm{LiFePO} 4$ battery in electric vehicles using multiple adaptive forgetting factors recursive least-squares. J. Power Sources 2015, 296, 215-224. [CrossRef]

21. Li, Y.; Wang, C.; Gong, J. A combination Kalman filter approach for state of charge estimation of lithium-ion battery considering model uncertainty. Energy 2016, 109, 933-946. [CrossRef]

22. Chaoui, H.; Gualous, H. Online parameter and state estimation of lithium-ion batteries under temperature effects. Electr. Power Syst. Res. 2017, 145, 73-82. [CrossRef] 
23. Liu, C.Z.; Liu, W.Q.; Wang, L.Y.; Hu, G.D.; Ma, L.P.; Ren, B.Y. A new method of modeling and state of charge estimation of the battery. J. Power Sources 2016, 320, 1-12. [CrossRef]

24. Feng, T.; Yang, L.; Zhao, X.; Zhang, H.; Qiang, J. Online identification of lithium-ion battery parameters based on an improved equivalent-circuit model and its implementation on battery state-of-power prediction. J. Power Sources 2015, 281, 192-203. [CrossRef]

25. Wei, Z.; Lim, T.M.; Skyllas-Kazacos, M.; Wai, N.; Tseng, K.J. Online state of charge and model parameter co-estimation based on a novel multi-timescale estimator for vanadium redox flow battery. Appl. Energy 2016, 172, 169-179. [CrossRef]

26. Xia, B.; Lao, Z.; Zhang, R.; Tian, Y.; Chen, G.; Sun, Z.; Wang, W.; Sun, W.; Lai, Y.; Wang, M.; et al. Online parameter identification and state of charge estimation of lithium-ion batteries based on forgetting factor recursive least squares and nonlinear Kalman filter. Energies 2017, 11, 3. [CrossRef]

27. Song, S.W.; Lim, J.S.; Baek, S.J.; Sung, K.M. Gauss newton variable forgetting factor recursive least squares for time varying parameter tracking. Electron. Lett. 2000, 36, 988-990. [CrossRef]

28. Shu-Hung, L.; So, C.F. Gradient-based variable forgetting factor RLS algorithm in time-varying environments. IEEE Trans. Signal Process. 2005, 53, 3141-3150. [CrossRef]

29. Paleologu, C.; Benesty, J.; Ciochina, S. A robust variable forgetting factor recursive least-squares algorithm for system identification. IEEE Signal Process. Lett. 2008, 15, 597-600. [CrossRef]

30. Lu, L.; Zhao, H.; Chen, B. Improved-variable-forgetting-factor recursive algorithm based on the logarithmic cost for volterra system identification. IEEE Trans. Circuits Syst. II Express Briefs 2016, 63, 588-592. [CrossRef]

31. Chu, Y.J.; Mak, C.M. A variable forgetting factor diffusion recursive least squares algorithm for distributed estimation. Signal Process. 2017, 140, 219-225. [CrossRef]

32. Chen, H.; Liu, H.J.; Li, D.L.; Dai, J. Time-varying parameters measurement by least square method with variable forgetting factors. High Volt. Eng. 2008, 34, 7.

33. Li, Q.; Wang, Y.; Zhang, X. Analysis and simulation of a variable forgetting factor RLS algorithm. Mod. Electron. Tech. 2008, 17. [CrossRef]

34. Haykin, S. Adaptive Filter Theory, 4th ed.; Prentice Hall: Upper Saddle River, NJ, USA, 2002.

35. Lee, S.S.; Kim, T.H.; Hu, S.J.; Cai, W.W.; Abell, J.A. Joining technologies for automotive lithium-ion battery manufacturing: A review. In Proceedings of the ASME 2010 International Manufacturing Science and Engineering Conference, Erie, PA, USA, 12-15 October 2010; pp. 541-549.

36. Schweighofer, B.; Raab, K.M.; Brasseur, G. Modeling of high power automotive batteries by the use of an automated test system. IEEE Trans. Instrum. Measur. 2003, 52, 1087-1091. [CrossRef]

37. Johnson, V.H. Battery performance models in advisor. J. Power Sources 2002, 110, 321-329. [CrossRef]

38. Johnson, V.H.; Pesaran, A.A.; Sack, T. Temperature-Dependent Battery Models for High-Power Lithium-Ion Batteries. In Proceedings of the 17th Annual Electric Vehicle Symposium, Montreal, QC, Canada, 16-18 October 2000.

(C) 2018 by the authors. Licensee MDPI, Basel, Switzerland. This article is an open access article distributed under the terms and conditions of the Creative Commons Attribution (CC BY) license (http://creativecommons.org/licenses/by/4.0/). 